\title{
MicroRNA-26a-5p inhibits proliferation, invasion and metastasis by repressing the expression of Wnt5a in papillary thyroid carcinoma
}

This article was published in the following Dove Press journal: OncoTargets and Therapy

\section{Dongliang Shi ${ }^{1}$ \\ Haiyan Wang ${ }^{2}$ \\ Mingjian Ding' \\ Meng Yang' \\ Chenhao Li' \\ Wenhua Yang' \\ Liang Chen'}

'Department of Surgical Oncology, Cangzhou Central Hospital, Cangzhou City, Hebei Province 061000, People's Republic of China; ${ }^{2}$ Department of Radiation Oncology, Cangzhou Central Hospital, Cangzhou City, Hebei Province 061000, People's Republic of China
Correspondence: Liang Chen Department of Surgical Oncology, Cangzhou Central Hospital, No. 16, Xinhua Road, Cangzhou City 061000, Hebei Province, People's Republic of China

Tel +863172075655

Email chenliangsy@sina.com
Background: Thyroid cancer (TC) is considered as the fastest growing malignancy in the human endocrine system, particularly papillary thyroid cancer (PTC). MicroRNAs (miRs) serve as a role in promoting or suppressing tumors in various types of malignant tumor including PTC. This study aims to explore whether microRNA-26a-5p (miR-26a-5p) could affect the proliferation, invasion and metastasis ability of PTC cells by regulating Wnt5a.

Materials and methods: The expression of miR-26a-5p was examined by qRT-PCR in PTC tissue samples (58 cases, mean age 53 years old) and PTC cell lines (K1 and BCPAP). Cell proliferation, invasion and migration were tested with CCK8 assay, colony formation assay, transwell invasion assay and wound healing assay, respectively. Luciferase reporting experiment was used to verify that Wnt5a is a molecular target of miR-26a-5p. The relationship between miR-26a-5p and Wnt5a was analyzed by qRT-PCR and Western blot and was further proved by Pearson's correlation analysis. Animal (24 nude mice) experiments were used to demonstrate that miR-26a-5p inhibits tumor growth by targeting Wnt5a.

Results: The expression of miR-26a-5p declined in PTC tissues $(P<0.01)$. The expression of miR-26a-5 was also significantly down-regulated in PTC tissues with advanced TNM stages $(P<0.01)$ and lymph node metastasis $(P<0.01)$ compared with normal thyroid tissues. Compared with normal human thyroid cell line Nthy-ori 3-1, the expression of miR-26a-5p in K1 cells and BCPAP cells were nearly 4.02-fold $(P<0.01)$ and 2.51-fold $(P<0.01)$ reduced. Up regulation of miR-26a-5p inhibited proliferation, colony formation, invasion and migration of PTC cells. MiR-26a-5p negatively regulated Wnt5a expression $(r=-0.887, P<0.01$ ), yet Wnt5a overexpression reversed the tumor-suppressive effect of miR-26a-5p in PTC. Animal experiments further verified that miR-26a-5p inhibited PTC growth by targeting Wnt5a.

Conclusion: Overexpression of miR-26a-5p depresses proliferation, invasion, metastasis of PTC via Wnt5a. Therefore, miR-26a-5p may represent a potentially effective target gene for PTC.

Keywords: papillary thyroid carcinoma, microRNA-26a-5p, Wnt5a, invasion, metastasis

\section{Introduction}

Thyroid malignancy is one of the common tumors in the endocrinology department with increasing incidence year by year in China, which is equivalent to almost $34 \%$ of the morbidity of the global thyroid cancer (TC) and nearly $2 \%$ of all newly diagnosed malignant tumors in the world. ${ }^{1-3}$ Papillary thyroid carcinoma (PTC), the most ordinary pathological type of thyroid carcinoma, is more than $90 \%$ of all thyroid malignancies. ${ }^{4}$ PTC, with a low-degree malignancy and a good prognosis, 
belongs to differentiated thyroid carcinoma. Surgical resection is the most important treatment for thyroid carcinoma, followed by $\mathrm{I}^{131}$ therapy, thyroxine endocrine therapy and radiotherapy. ${ }^{5,6}$ However, there are still a small number of patients who have invasion of the surrounding tissues, lymph node metastasis or even distant metastasis in the early stage of thyroid cancer, or recurrence and metastasis after surgery. Therefore, recurrence and metastasis are still the main causes of PTC treatment failure and death. ${ }^{7,8}$ There is increasing evidence that abnormal activation and dysfunction of key genes can lead to the progression of PTC. ${ }^{9,10}$ Therefore, investigation of molecular markers of PTC plays a crucial role in improving its therapeutic strategy and prognosis.

The Wnts contain a variety of secretory glycoproteins that control vital developmental processes including cell differentiation, proliferation and migration. ${ }^{11}$ Previous studies have revealed that the progression of various malignant tumors is affected by the abnormal activation of the Wnt pathway. ${ }^{12,13} \mathrm{Wnt5a}$, an important signaling molecule in the non-canonical Wnt family, has been involved in nearly all parts of the non-canonical Wnt pathway. ${ }^{14,15}$ The latest experiments show that Wnt5a plays a key role in the progression of various malignancies, but different studies show that the biological significance of Wnt5a in a variety of malignant tumors is not consistent. ${ }^{16}$

MicroRNAs (miRs) consist of 18-25 nucleotides and belong to a kind of non-coding RNAs. They generally regulate the target $\mathrm{mRNAs}$ by incompletely or completely binding to the 3 '-untranslated region (UTR) of the target mRNA, ${ }^{17,18}$ resulting in degradation of mRNA or depressing protein translation. Previous reports have shown that mRNAs play important roles in multiple tumor-related biological processes, comprising differentiation, proliferation, metastasis and apoptosis. ${ }^{19,20}$ In recent years, researchers have found abnormal expression of various miRs in PTC, which suggests that these miRs serve important roles in carcinogenesis. ${ }^{21}$ MicroRNA-26a-5p (miR$26 \mathrm{a}-5 \mathrm{p}$ ), a tumor-associated $\mathrm{miR}$, has been found to be implicated in the development of various cancers by regulating tumor cell proliferation, invasion, migration and apoptosis. $^{22}$ Based on bioinformatic analysis of RNA-sequencing data, Lu et al believed that miR-26 was implicated in the molecular mechanism of thyroid cancer occurrence and development, ${ }^{23}$ but the particular function and regulatory mechanism of miR-26a-5p in PTC or other subtypes remain unclear. In this experiment, we not only compared the expression level of miR-26a-5p in PTC and normal thyroid tissues but also demonstrated the specific functions of miR-26a-5p in vitro and in vivo. MiR-26a-5p has been shown to target Wnt5a directly. These findings suggested that miR-26a-5p may become a novel target for PTC.

\section{Materials and methods Tissue samples and cell lines}

Fifty-eight paired PTC and their corresponding near normal tissues excised from patients (with 15 men and 43 women, mean age 53 years old) were gathered from Department of Surgical Oncology, Cangzhou Central Hospital (Cangzhou, China) between January 2014 and June 2014. All samples were pathologically diagnosed by an expert pathologist. Surgically removed tissue samples were frozen rapidly, and then stored at $-80^{\circ} \mathrm{C}$ until the experiment started. The Institutional Ethics Committee of Cangzhou Central Hospital approved the research protocol, which was conducted in accordance with the Declaration of Helsinki. All patients provided written informed consent. Human normal thyroid follicular epithelial cell line Nthy-ori-3-1 and PTC cell lines K1 as well as BCPAP were purchased from the American Type Culture Collection (ATCC, USA) and we cultured these cell lines in DMEM (Thermo Fisher Scientific, Waltham, MA, USA) supplemented with $10 \%$ fetal calf serum in a $5 \%$ carbon dioxide atmosphere at $37^{\circ} \mathrm{C}$.

\section{Quantitative real-time PCR (qRT-PCR) assay}

We used the Trizol reagent (Invitrogen) to extract total RNA from tissues and cells in accordance with the instructions. We used a TaqMan MiRNA Reverse Transcript Kit (Applied Biosystems, Foster City, CA, USA) with a specific stem-loop primer for reverse transcription. Applied Biosystems Prism7900 Fast Sequence Detection System using TaqMan miRNA primers (Applied Biosystems) was performed for PCR reactions. We detected the expression levels of Wnt5a mRNA and miR-26a-5p by Western blot and qRT-PCR, and GAPDH and U6 were used as endogenous control, respectively. The qRT-PCR was performed under the following conditions: $95^{\circ} \mathrm{C}$ for $2 \mathrm{mins}$, then $95^{\circ} \mathrm{C}$ for $10 \mathrm{~s}, 55^{\circ} \mathrm{C}$ for $1 \mathrm{~min}$, $72^{\circ} \mathrm{C}$ for $1 \mathrm{~min}$. We used the $2^{-\Delta \Delta \mathrm{Ct}}$ method to test the relative expression levels. The primers used were as follows: 
miR-26a-5p-forward: 5'-UCCAUAAAGUAGGAAAC ACUACA-3',

backward: 5'-CAGUACUUUUGUGUAGUACAA-3'. Wnt5a-forward: 5'-ACCACATGCAGTACATCGGA G-3',

backward: 5'-GAGGTGTTATCCACAGTGCTG-3'. GAPDH-forward: 5'-GAAGGTGAAGGTCGGAGTC-3', backward: 5'- GAAGATGGTGATGGGATTTC-3'. U6-forward: 5'-CTCGCTTCGGCAGCACATATACT-3', backward: 5'-ACGCTTCACGAATTTGCGTGTC-3'.

\section{Cell transfection}

We purchased miR-26a-5p mimics (miR-26a-5p) and their corresponding negative control (miR-NCs) as well as Wnt5a over expression plasmid vector (Wnt5a) which were synthesized by Genechem Company (Shanghai, China). DNA alignment analysis verified all of these. The reagent of Lipofectamine 2000 (Invitrogen, Carlsbad, USA) was used to transfect cells following the instructions. We analyzed gene expression after $24 \mathrm{hrs}$ of transfection, then carried out cell proliferation, cloning, migration and invasion experiments.

\section{Western blot analysis}

We used ice-cold RIPA lysis buffer supplemented with protease inhibitor cocktail and phenylmethanesulfonyl fluoride (PMSF) to extract the total protein. Furthermore, we mixed cell lysates with loading buffer which subjected in $10 \%$ sodium dodecyl sulfate-polyacrylamide gel electrophoresis, followed by being transferred onto polyvinylidene difluoride (PVDF) membranes. We used 5\% skimmed milk to block the membranes for $2 \mathrm{hrs}$, and then used antibodies against Wnt5a (Abcam, Cambridge, UK) to incubate at $4{ }^{\circ} \mathrm{C}$ overnight. We used TBST to wash the membranes for 3 times and then used the corresponding horseradish peroxidase-conjugated secondary antibodies (Cell Signaling Technology, Boston, USA) to incubate for another $1 \mathrm{hr}$ at $37^{\circ} \mathrm{C}$. We used an enhanced ECL Chemiluminescence kit (UltraSignal, Beijing, China) according to the instructions to visualize the proteins. We exposed the signals to X-ray films and set up GAPDH as the loading control.

\section{Cell proliferation assay}

We used a Cell Counting Kit-8 (CCK-8, Dojindo, Japan) according to the manufacturer's protocol to measure cell proliferation. In brief, we inoculated transfected cells of logarithmically growing $\left(5 \times 10^{3}\right.$ cells/well $)$ into 96 -well culture plates, and then cultured these cells for 24-96 hrs. We added the CCK-8 assay solution $(10 \mu \mathrm{L})$ to every well within the prescribed time. We used an enzyme immunoassay analyzer (Thermo Fisher Scientific, Inc., USA) to measure the absorbance at $450 \mathrm{~nm}$.

\section{Colony formation assay}

We did plate colony formation assay to assess cell colony formation ability. We added K1 cells with stable expression of miR-26a-5p or miR-NC to every well (100 cells/ well) of a 6-well plate and incubated these cells for approximately 2 weeks depending on instructions. Then, we gently washed the plate and stained it with $0.2 \%$ crystal violet. We calculated the number of colonies by observing the growth of the single cell.

\section{Transwell invasion assay}

We performed transwell invasion assay to determine cell invasion. Briefly, we seeded cells in 24-well plates without serum medium but containing matrigel-coated insert filters. We used culture medium with $30 \%$ FBS to filled the lower chamber. We fixed cells and stained them with $0.1 \%$ crystal violet (Sigma-Aldrich) after incubation for 48 hrs at $37^{\circ} \mathrm{C}$. We quantitatively analyzed the cells invading the matrigel membrane.

\section{Wound healing assay}

For wound healing assay, we seeded transfected cells in $60 \mathrm{~mm}$ dishes and cultured them in DMEM for $24 \mathrm{hrs}$. We wounded cell layers using a $10 \mu \mathrm{L}$ tip to make a gap, and then cultured them without serum DMEM for additional $24 \mathrm{hrs}$. We used an optical microscope with $200 \times$ magnification to photograph the wounded gaps at 0 and $24 \mathrm{hrs}$. We measured and analyzed the gap distances of migrating cells from 5 different areas for every wound.

\section{Luciferase reporter assays}

We constructed the Wnt5a 3'UTR luciferase reporter gene plasmid by using luciferase reporter gene plasmid pMIR. We subcloned wild-type Wnt5a3'UTR or its corresponding binding site-deleted miR-26a-5p into the pMIR plasmid. Luciferase reporter gene was detected by pmirGlo Luciferase Detection System (Promega, Madison, USA) in 96-well plates. We used Lipofectamine 2000 (Invitrogen, Carlsbad, USA) to co-transfected cells with $10 \mu \mathrm{g}$ of Wnt5a-wt and its corresponding binding site-deleted 
miR-26a-5p or miR-26a-5p mimics. We used a DualLuciferase Reporter Assay kit (Promega) to measure luciferase activity according to the manufacturer's instructions after 48 hrs. We made use of SpectraMax M5 instrument software (Molecular devices, San Francisco, USA) to analyze the results. We repeated each test 3 times.

\section{Xenograft tumor model studies}

Twenty-four healthy nude mice of similar weight were obtained from the Laboratory Center of Cangzhou Central Hospital. We randomly divided them into 2 groups $(n=12$ in each group) which were subcutaneously injected K1 cells stably expressing miR-26a-5p or miR-NC, respectively. We measured the width (W) and length (L) of the tumors to observe the proliferation of the tumors, and then calculated the volume $(\mathrm{V})$ of the tumors according to the formula: $\mathrm{V}=\left(\mathrm{L} \times \mathrm{W}^{2}\right) / 2$. After 6 weeks, all these animals which were injected were subject to death. All these animals were sacrificed after 42-days' injection. The tumors were excised, weighed, and then quickly stored at $-80^{\circ} \mathrm{C}$ for further assay. These experiments were approved by the Animal Care and Utilization Committee of Cangzhou Central Hospital and the protocols complied with the guidelines for the welfare and use of animals in cancer research.

\section{Statistical analysis}

SPSS 24.0 software (IBM, Chicago, IL, USA) was applied to statistical analysis. Student's $t$-test was performed to analyze two groups and one-way analysis of variance (ANOVA) with Tukey's post-hoc test was performed to analyze multiple groups. The Mann-Whitney U test was performed to determine the differences between thyroid carcinoma and paired adjacent noncancerous tissues in the expression of miR-26a-5p and Wnt5a. Pearson's correlation analysis was used to assess the relationship between the expression of miR-26a-5p and Wnt5a. $P$-value $<0.05$ was considered to show a statistically significant difference.

\section{Results}

The expression level of miR-26a-5p in PTC tissues and cells was decreased

In order to clarify the biological role of miR-26a-5p, we first explored the expression level of miR-26a-5p in PTC tissues and adjacent normal tissues in 58 patients suffering from PTC by qRT-PCR assay and analyzed by MannWhitney $U$ test. The results of qRT-PCR assay demonstrated that the expression of miR-26a-5p in PTC was less than that in normal thyroid tissues $(P<0.01)$ (Figure 1A). To further discuss the clinical significance of miR-26a-5p in PTC, the relationship between expression levels of miR-26a-5p and clinicopathological feature was analyzed by Student's $t$-test. The median expression level of miR-26 (0.565) was used as the cutoff value. These 58 PTC patients were divided into lower expression group $(n=25)$ and higher expression group $(n=33)$ according to the cutoff value of miR-26a-5p. It was noted that the expression of miR-26a-5p was down regulated in advanced TNM stages $(P<0.01)$ and PTC tissues with lymph node metastasis $(P<0.01)$ (Table 1 , Figure $1 \mathrm{~B}$ and C). In addition, the expression of miR-26a-5p was detected in various different thyroid carcinoma cell lines and was analyzed by Student's $t$-test. The results showed that the expression of miR-26a-5p in K1 cells and in BCPAP cells was about 4.02-fold $(P<0.01)$ and 2.51-fold $(P<0.01)$ reduction compared with normal human thyroid cell line Nthy-ori-3-1, respectively (Figure 1D). The data above suggested that miR-26a-5p may be a suppressor to inhibit the tumorigenesis of PTC.

\section{MiR-26a-5p suppressed cell proliferation, colony formation, invasion and metastasis in PTC}

To further speculate the probable biological function of miR-26a-5p in PTC, we used miR-26a-5p mimics to perform the gain function analysis and carried out Student's $t$ test to calculate relevant data. As shown in Figure 2A, compared with miR-NC group, transfection of miR-26a-5p mimics resulted in about 3.98-fold increase of miR-26a-5p expression in miR-26a-5p mimic group $(P<0.01)$. Overexpression of miR-26a-5p down-regulated proliferation of PTC cells which was confirmed by CCK 8 assay $(P<0.01)$ (Figure 2B). Meanwhile, the increase of miR-26a-5p significantly declined the number of colonies of K1 cells, suggesting that miR-26a-5p depressed the colony-forming ability in PTC (Figure 2C).

We further investigated the impact of miR-26a-5p on invasion and metastasis of PTC cells. It was shown that transfection of miR-26a-5p mimics brought about 2.19fold reduction in the number of cells passing through the basement membrane compared with miR-NC group, which suggested that ectogenic miR-26a-5p repressed the invasive ability of PTC cells $(P<0.01)$ (Figure 2D). Similar to the result earlier, wound healing assay demonstrated 

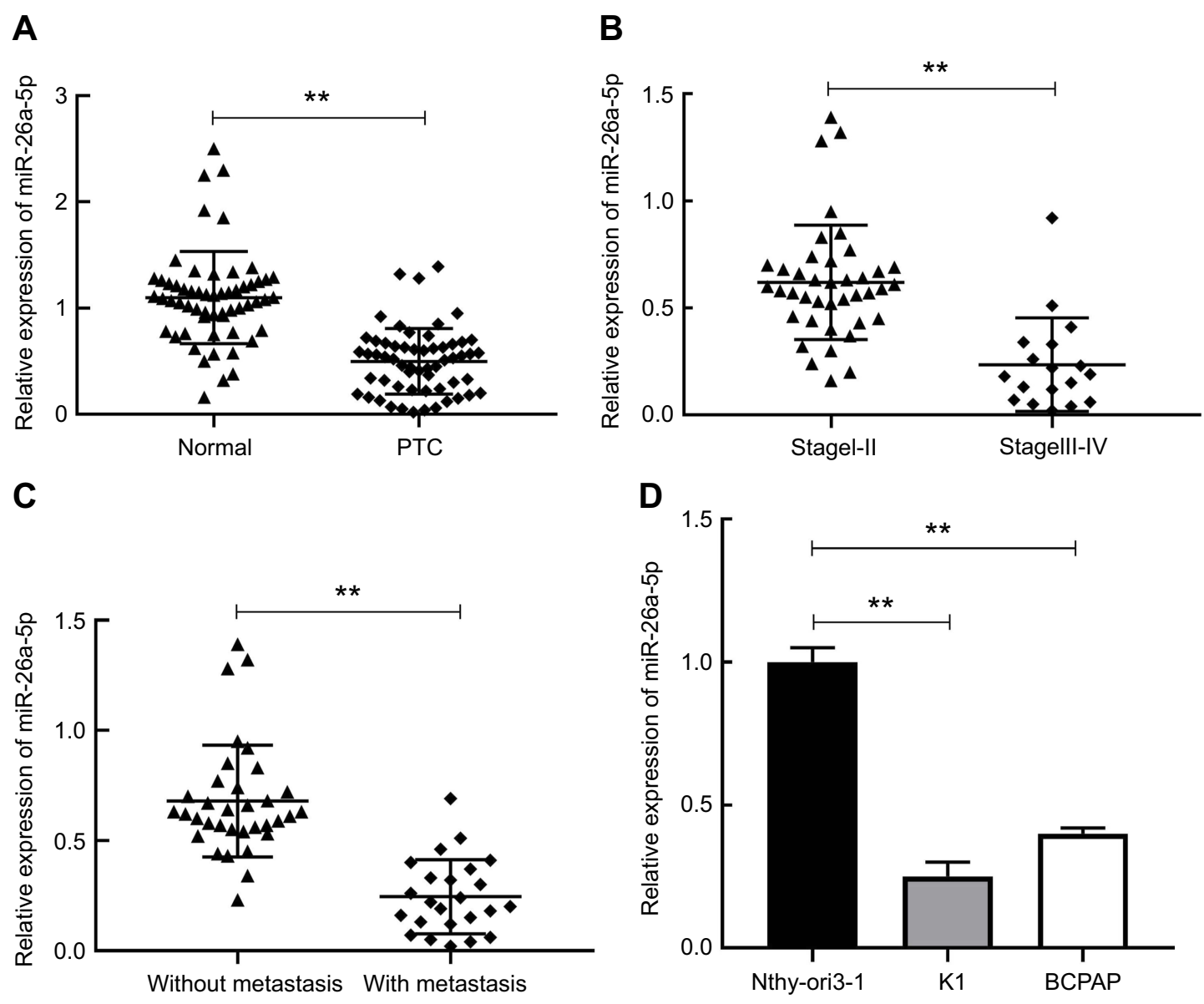

Figure I The expression level of miR-26a-5p in PTC tissues and cells was decreased. (A) The relative expression of miR-26a-5p in PTC tissues and corresponding adjacent normal tissues was measured using qRT-PCR. (B-C) The relative expression of miR-26a-5p in PTC tissues with different TNM stages and with or without lymph node metastasis. (D) The relative expression of miR-26a-5P in two thyroid cancer cell lines KI, BCPAP and thyroid gland epithelial cell line Nthy-ori-3-I was detected by qRT-PCR. $* * P<0.01$.

Abbreviation: PTC, papillary thyroid carcinoma.

that transfection of miR-26a-5p mimics caused nearly 2.03 -fold reduction in the number of cells migration compared with miR-NC group, which revealed ectogenic miR26a-5p abrogated the migration of PTC cells $(P<0.01)$ (Figure 2E). These experiments in vitro suggest that miR-26a-5p may effectively depress the growth, invasion and migration of PTC cells.

\section{Wnt5a is a target gene of miR-26a-5p that is predicted in PTC}

We used TargetScan (http://www.targetscan.org/vert_71/), mi-Randa (http://www.microrna.org/microrna/ getGeneForm.do) and PicTar (https://pictar.mdc-berlin.de) databases to predict potential targets for regulating PTC cell proliferation, invasion and metastasis. Wnt5a seems to be the most suitable candidate target gene for miR-26a-5p for it was found that Wnt5a is associated with poor prognosis in PTC in our previous study. ${ }^{24}$ To further examine whether miR-26a-5p could target Wnt5a and the underlying molecular mechanism, 3'-untranslated regions reporter vectors (WT, Wnt5a 3'-UTR) comprising the forecasted alignments were constructed. MiR-26a-5p mimics significantly decreased the relative firefly luciferase activity by nearly 3.90-fold that was demonstrated by luciferase activity assay, which did not occur when the predicted corresponding binding sites were deleted (Figure $3 \mathrm{~A}$ and $\mathrm{B}$ ). Both the mRNA and the protein expression level of Wnt5a were remarkably declined in TPC cell lines K1 after transfected with miR-26a-5p mimics those were revealed by qRT-PCR and Western blot results, and Student's $t$-test was used for statistical analysis (Figure 3C and D). Wnt5a mRNA expression was also detected by qRT-PCR in 58 pairs of PTC tissues and the corresponding normal tissues, respectively, and data were analyzed by Mann-Whitney U test. As Figure 3E showed, comparing to normal thyroid tissues, Wnt5a mRNA expression was found to be obviously up 
Table I Correlation between clinicopathological features and miR-26a-5p expression in 58 patients with PTC

\begin{tabular}{|c|c|c|c|c|}
\hline \multirow[t]{2}{*}{ Variables } & \multirow[t]{2}{*}{ Cases } & \multicolumn{2}{|c|}{ miR-26 expression } & \multirow[t]{2}{*}{$P$ value } \\
\hline & & High & Low & \\
\hline Age(years) & & & & 0.147 \\
\hline$<45$ & 20 & 11 & 9 & \\
\hline$\geq 45$ & 38 & 14 & 24 & \\
\hline Gender & & & & 0.489 \\
\hline Male & 15 & 7 & 8 & \\
\hline Female & 43 & 18 & 25 & \\
\hline TNM stage & & & & 0.001 \\
\hline I-II & 40 & 23 & 17 & \\
\hline III-IV & 18 & 2 & 16 & \\
\hline $\operatorname{Size}(\mathrm{cm})$ & & & & 0.542 \\
\hline$<1$ & 41 & 18 & 23 & \\
\hline$\geq 1$ & 17 & 7 & 10 & \\
\hline Lymph node metastasis & & & & 0.004 \\
\hline Yes & 24 & 5 & 19 & \\
\hline No & 34 & 20 & 14 & \\
\hline Multifocality & & & & 0.311 \\
\hline Yes & 20 & 10 & 10 & \\
\hline No & 38 & 15 & 23 & \\
\hline
\end{tabular}

regulated in the PTC tissues. The expression levels of miR26a-5p were negatively correlated with the expression levels of Wnt5a mRNA in PTC tissues that was revealed by Pearson's correlation analysis $(\mathrm{n}=58, \mathrm{r}=-0.887, P<0.01)$ (Figure 3F). The hypothesis that miR-26a-5p may directly target Wnt5a in PTC was proved by these data.

\section{The tumor-suppressive effect of miR-26a-5p in PTC was reversed by up regulation of $\mathrm{Wnt} 5 \mathrm{a}$}

To investigate the functional correlation between Wnt5a and miR-26a-5p, we further co-transfected the miR-26a-5p plasmid and Wnt5a up regulated plasmid (without 3'-UTR) into TPC cell lines K1, and the data were analyzed by ANOVA test. We observed that the expression of Wnt5a which was decreased by the over expression of miR-26a-5p was restored in TPC cell lines $\mathrm{K} 1$ transfected with the Wnt5a over expression plasmid (Figure 4A and B). Furthermore, the miR-26a$5 p$-mediated suppressive effect concerning cell proliferation, colony formation, invasion and migration in TPC cell lines K1 was partially rescued by up regulation of Wnt5a (Figure 4C-F). These data revealed that
miR-26a-5p suppressed PTC cell growth, invasion and migration by up regulating Wnt5a expression.

\section{Tumor growth was suppressed by miR- $26 a-5 p$ in vivo}

To verify the function of miR-26a-5p in vivo, the animal experiment was performed. We created tumor xenograft mouse models by subcutaneously injecting $\mathrm{K} 1$ cells steadily expressing miR-NC or miR-26a-5p. Tumor sizes were measured every week until the mice were put to death. We used Student's $t$-test to make statistical analysis. The results showed that tumor growth was faster in the miR$\mathrm{NC}$ group compared with that in the miR-26a-5p group (Figure 5A). The mice were executed and the tumors were taken out and weighed after 6 weeks' injection. Comparing with the miR-26a-5p group, these neoplasms size and weight (Figure 5B and C) in the miR-NC group were obviously increased. The expression of miR-26a-5p was increased in the group of miR-26a-5p (Figure 5D), while the expression of Wnt5a was reduced (Figure $5 \mathrm{E}$ and F). These data showed that PTC tumor growth was inhibited by miR-26a-5p in vivo via suppressing Wnt5a expression.

\section{Discussion}

Thyroid malignancy, a common endocrine neoplasm, is rapidly increasing around the world. ${ }^{4}$ PTC, as the most common type of TC, is characterized by a low degreemalignancy, well clinical prognosis, but early lymph node metastasis. The oncogenesis of PTC is closely relevant to the genetic changes in thyroid cells, and the deletion or activation of one or more genes may cause the malignant proliferation of cells. ${ }^{25,26}$ Therefore, it is an urgent clinical problem to seek potentially effective target gene locus for patients with metastasis and postoperative recurrence.

Previous studies have confirmed that microRNAs (miRs) are implicated in the malignant evolution of PTC. ${ }^{27}$ MiRs can modulate a wide variety of targets, which make it possible to assume that a solitary miR via targeting many genes adjust tumor evolution in various steps. ${ }^{28}$ MiRs, consisting of short-chain non-coding RNAs, are a kind of post-transcriptional regulators and can lead to the silencing of target genes by combining the complementary alignment in the 3 '-untranslated region ( 3 '-UTR) of a variety of mRNA transcripts. ${ }^{20,29}$ Current studies have proved that miRs play a role as oncogene or tumor 
A

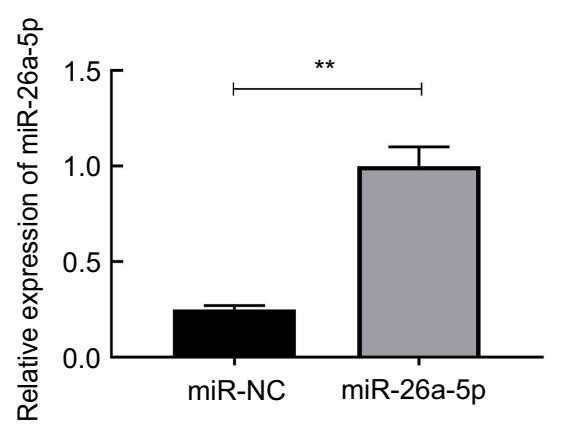

C

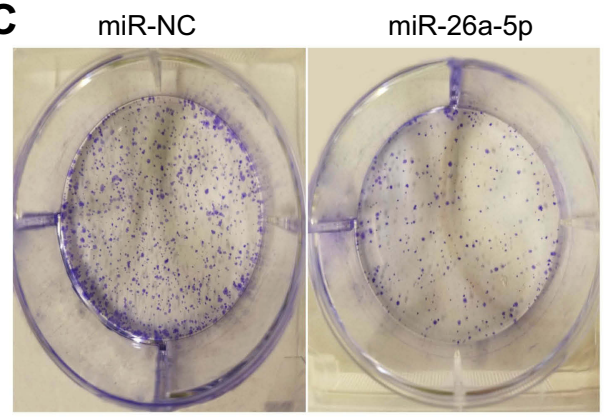

D

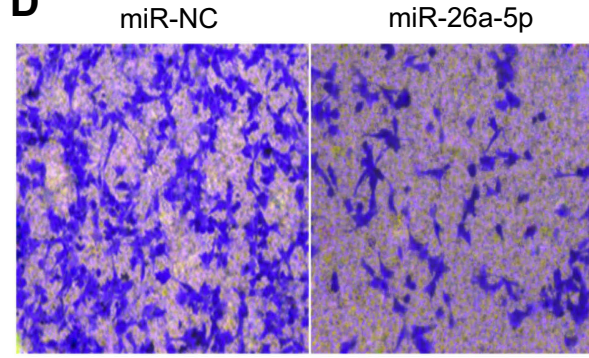

E
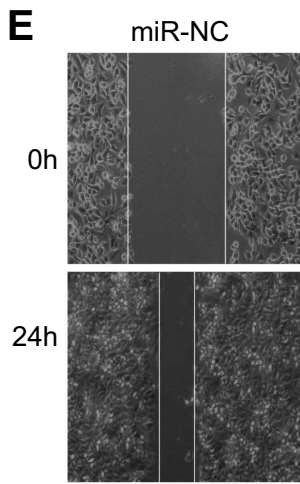

miR-26a-5p
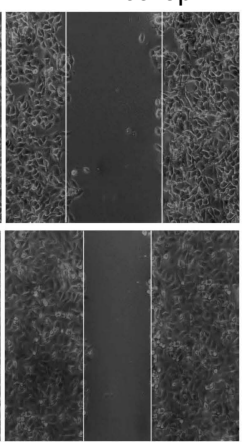

B
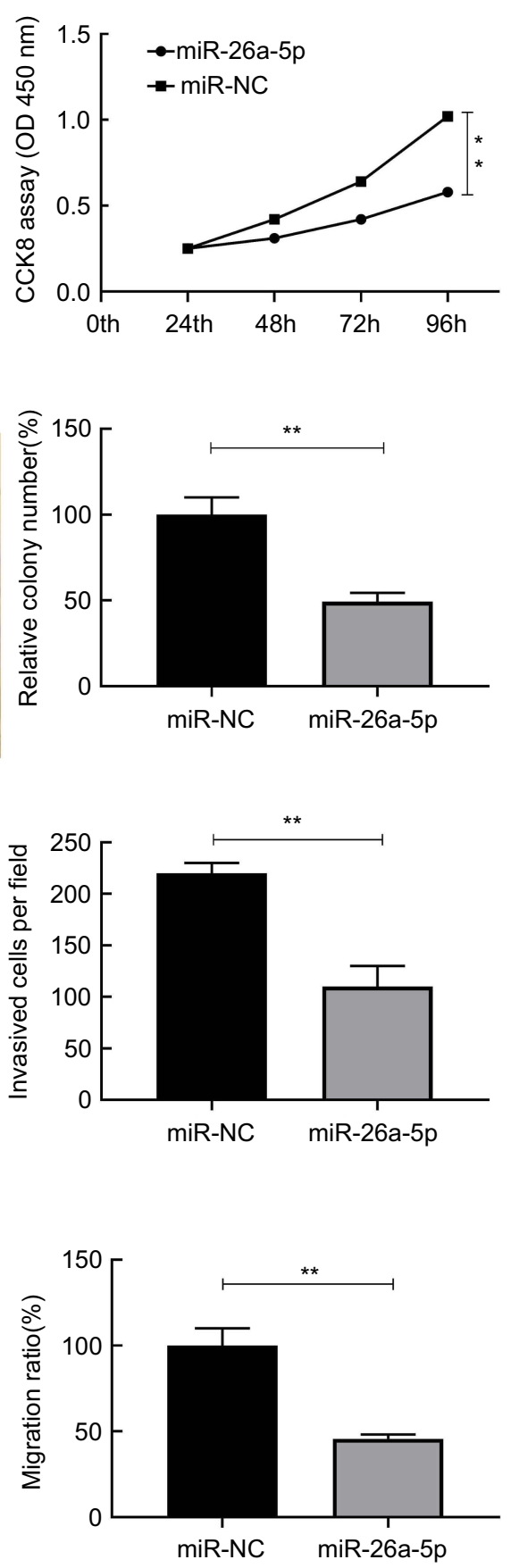

Figure 2 MiR-26a-5p suppressed cell proliferation, colony formation, invasion and metastasis in PTC. (A) MiR-26a-5p expression in KI cells transfected with miR-26a-5p and miR-NC mimics. (B-E) The effect of miR-26a-5p on the proliferation, colony formation, migration and invasion of KI cells were examined, respectively, by CCK8 assay, colony formation assay, wound healing assay and the transwell invasion assay. $* * P<0.01$.

Abbreviation: PTC, papillary thyroid carcinoma.

suppressor in malignant tumors. ${ }^{30,31}$ It has been confirmed that miR-221, miR-155 and miR-222 can promote the formation and evolution of thyroid carcinoma, ${ }^{32-34}$ while miR-141 and miR-7 repress the neoplasia and development of thyroid malignancy. ${ }^{35,36}$ MiR-26 was first cloned from Hela cells and its expression had no tissue specificity. Current reports have found that miR-26 serves as a critical role in bone injury repair, osteogenic 
A
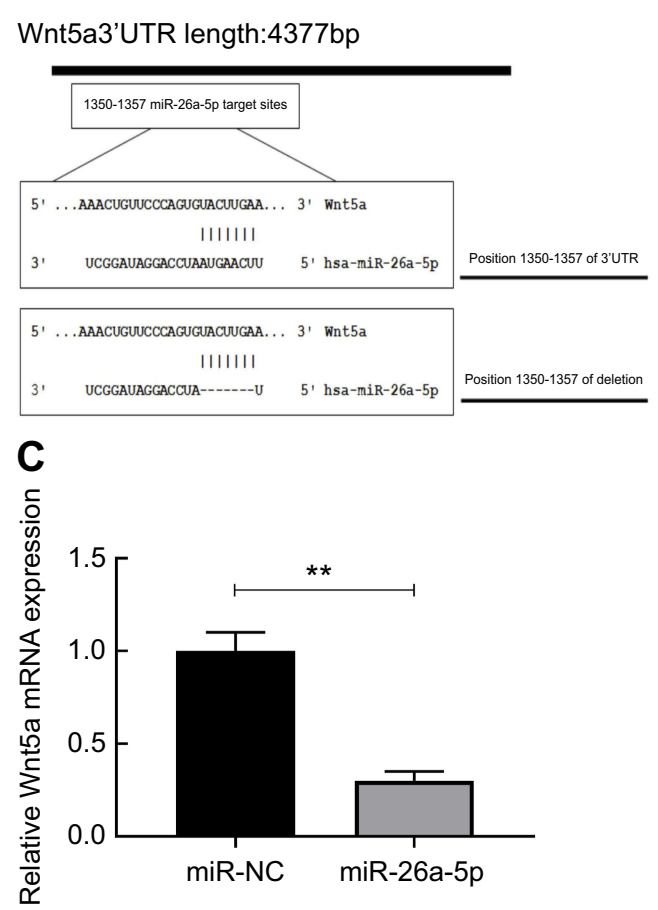

E

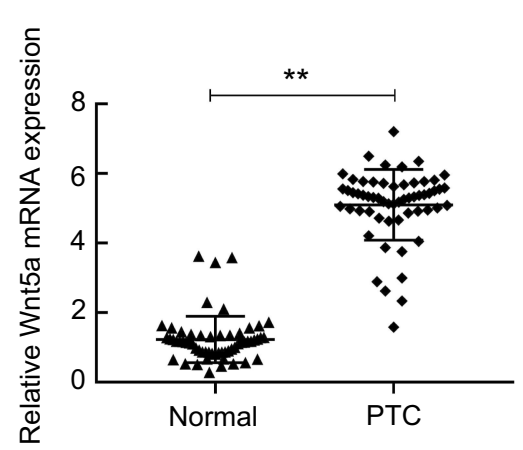

B

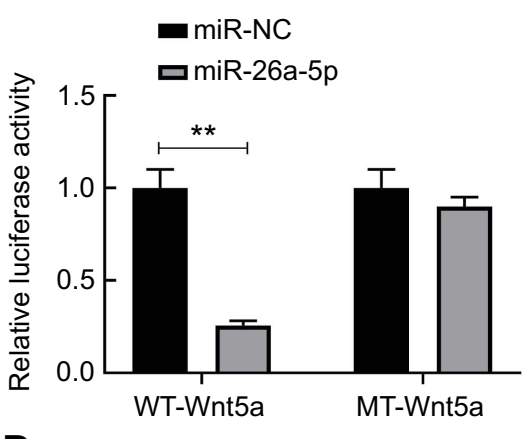

D

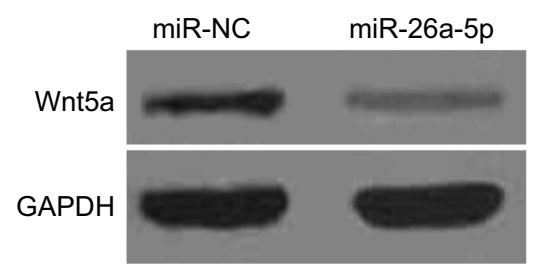

$\mathbf{F}$

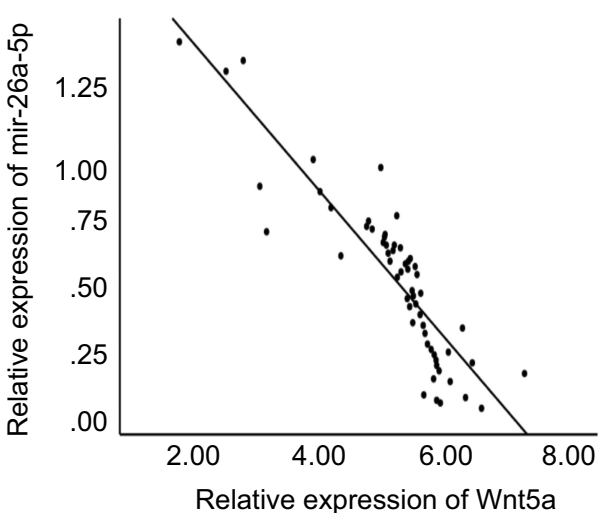

Figure $3 \mathrm{Wnt5a}$ is a target gene of miR-26a-5p that is predicted in PTC. (A) Sequence alignment of putative wild-type (WT) and deleted miR-26a-5p corresponding binding sites in the 3'UTR of Wnt5a was indicated. (B) Relative luciferase activities were analyzed in KI cells co-transfected with the pmiR-Wnt5a-WT or pmiR-Wnt5a-MT reporter plasmid, and miR-26a-5p or miR-NC plasmid. (C-D) Wnt5a expression on mRNA and protein level was determined in KI cells transfected with miR-26a-5p or miR-NC. (E) mRNA expression of Wnt5a was determined in PTC tissues compared with the corresponding adjacent normal tissues. (F) Wnt5a mRNA levels were inversely correlated with the miR-26a-5p level in 58 PTC samples using Pearson's correlation analysis. $* * P<0.01$.

Abbreviations: PTC, papillary thyroid carcinoma; MT, mutant type.

differentiation, diabetic wound healing and lipid metabolism. Recent studies have further confirmed that miR-26 is involved in the formation and evolution of neoplasm. Moreover, miR-26 plays opposite roles in the pathogenesis of different tumors. MiR-26 presents tumor-suppressive effect in some tumors, such as esophageal squamous cancer, gastric cancer and bladder cancer, ${ }^{37-39}$ while in other tumors miR-26 exhibits carcinogenic effect, for example, glioma ${ }^{40}$ cholangiocarcinoma. ${ }^{41} \mathrm{Lv}$ et al pointed out that miR-26 inhibits PTC tumorigenesis and progression by targeting the expression of CKS2. ${ }^{42}$ Gong et al thought that down regulated expression of miR-26 can promote growth of PTC cells and increase the sensitivity of PTC cells to TMX by depressing the expression of ARPP-19. ${ }^{43}$ However, the role of miR-26 in PTC and the detailed regulatory mechanisms modulating the malignant progression of PTC cells are not fully elucidated.

Previous studies have confirmed that Wnt5a usually activates the following two signal transduction pathways: $\mathrm{Wnt} / \beta$-catenin passageway and $\mathrm{Wnt} / \mathrm{Ca}^{2+}$ passageway. At 
A

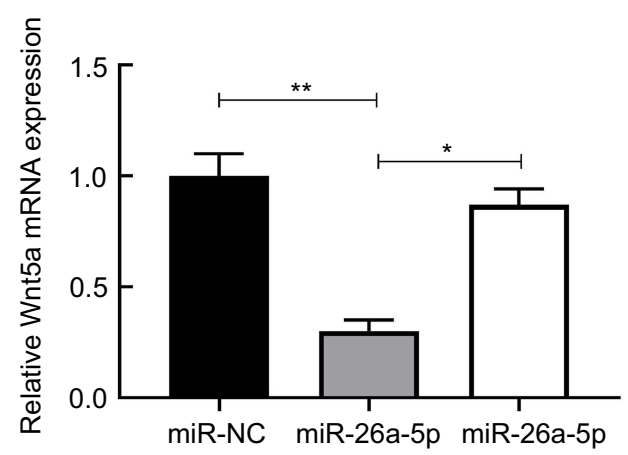

$+$

C

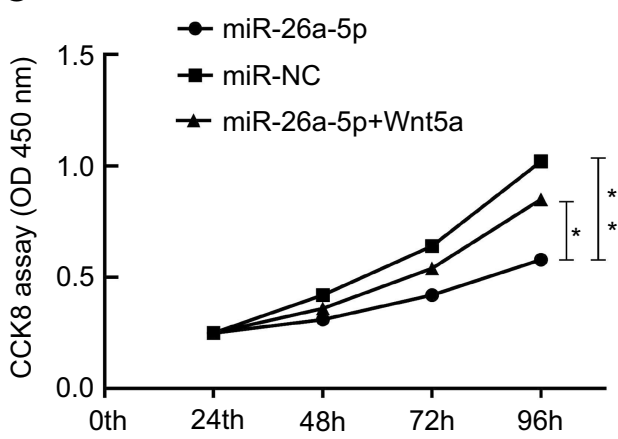

E

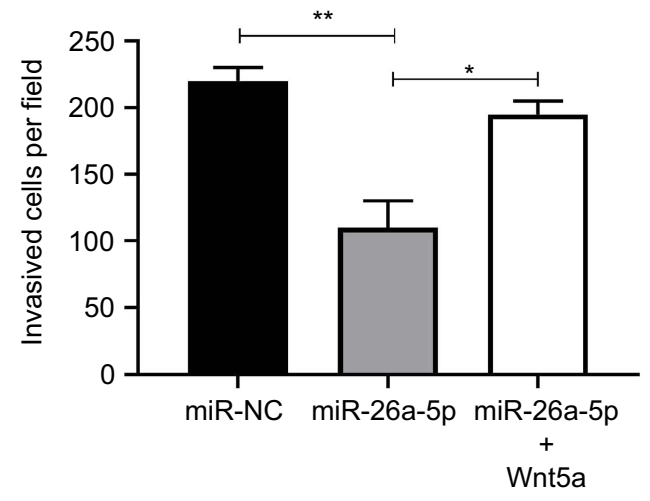

B

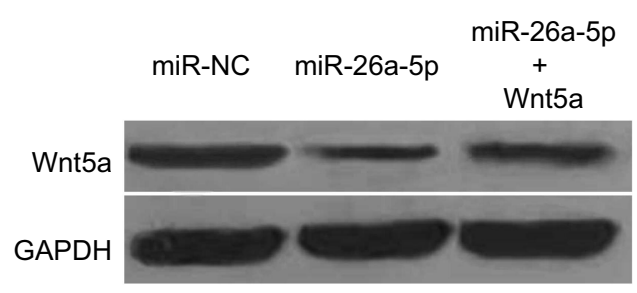

D

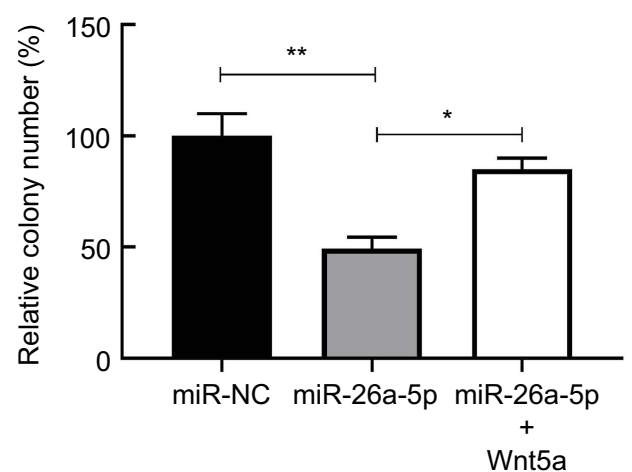

$\mathbf{F}$

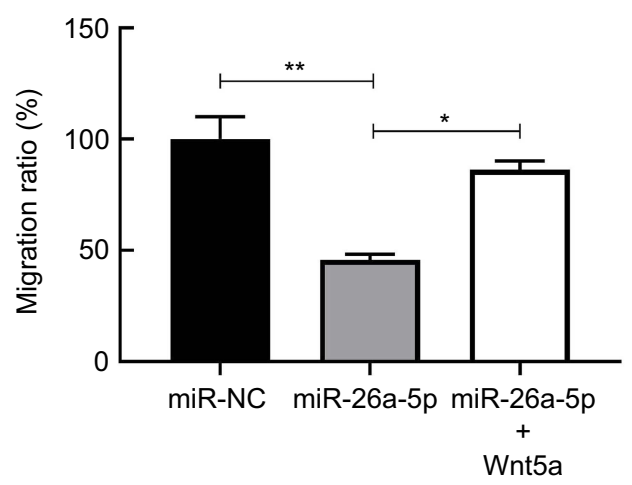

Figure 4 The tumor-suppressive effect of miR-26a-5p in PTC was reversed by Wnt5a up regulated. (A-B) Wnt5a expression on mRNA and protein levels was determined in $\mathrm{KI}$ cells transfected with the miR-26a-5p or miR-NC plasmid, and with or without the Wnt5a over expression plasmid (without 3 'UTR). (C-F) Over expression of Wnt5a partially rescued miR-26a-5p-mediated inhibitory effect on cell proliferation, colony formation, migration and invasion in $\mathrm{KI}$ cells. $* P<0.05$, $* * P<0.01$.

Abbreviation: PTC, papillary thyroid carcinoma.

present, the research on $\mathrm{Wnt} / \beta$-catenin is relatively distinct, while the $\mathrm{Wnt} / \mathrm{Ca}^{2+}$ pathway is not very clear. ${ }^{44,45}$

Abnormal Wnt signal transduction has become a critical mechanism in the etiopathogenesis of some cancers. ${ }^{46,47}$ Wnt5a activates classical or non-classical Wnt signaling pathways through different receptors and inhibits Wnt/ $\beta$ catenin pathways through non-classical signaling pathways. Wnt5a can both inhibit and activate the Wnt/ $\beta$-catenin signaling pathway, which seems to indicate that Wnt5a has a paradoxical role in cancer. ${ }^{15}$ Related studies have shown that Wnt5a has the role of tumor initiation and tumor inhibition in different malignant tumors. For example, over-expression of Wnt5a has been found in prostate, pancreas, stomach and lung carcinoma, ${ }^{48}$ while Wnt5a showed the opposite effect in some other cancers. The expression of Wnt5a is down-regulated in leukemia and neuroblastoma. ${ }^{49,50}$ Therefore, Wnt5a acts as a tumor inhibitor. In addition, Wnt5a can be regulated by a variety of miRs and plays different roles in multiple 
A

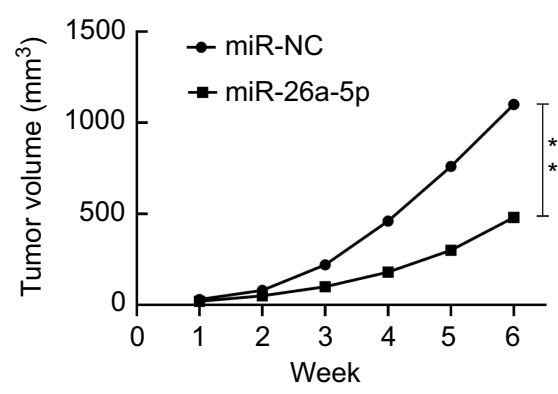

C

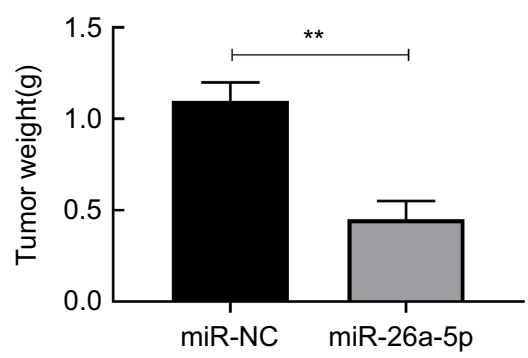

E

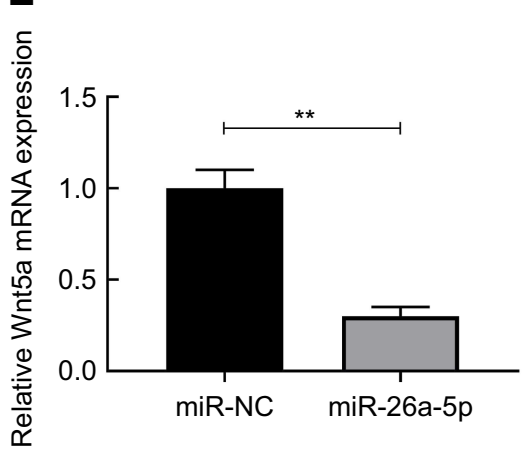

B

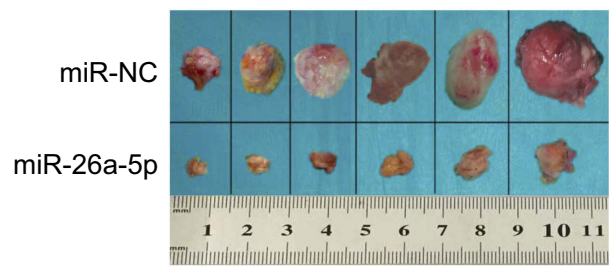

D

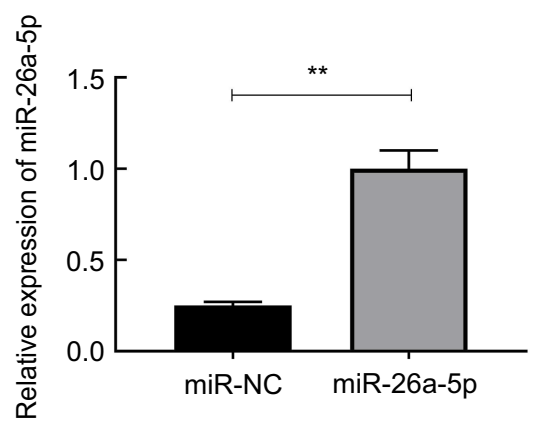

$\mathbf{F}$

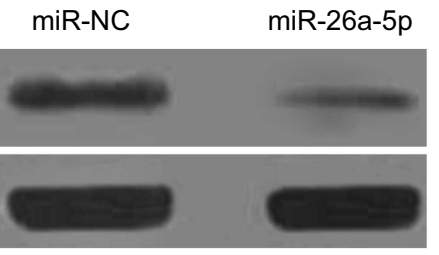

Figure 5 Tumor growth was suppressed by miR-26a-5p in vivo. (A) Tumor volume was measured every week after injected with KI cells with stable expression of miR-26a$5 p$ or miR-NC. (B) Tumor tissues were imaged after the mice were sacrificed. (C)The tumor weight was measured after the mice were sacrificed. (D) The expression of miR-26a-5p was detected in tumor tissues using qRT-PCR. (E-F) Wnt5a expression on mRNA and protein levels was measured in tumor tissues.**P<0.0I.

Abbreviation: NC, normal control.

tumor tissues. For example, miR-1253 inhibits proliferation and invasion of non-small cell lung cancer by regulating Wnt5a, ${ }^{51}$ miR-154 inhibits the occurrence and progression of osteosarcoma by targeting Wnt5a. ${ }^{52}$ As an important component of the Wnt family, the function of Wnt5a is not completely clear in thyroid carcinoma. Kremenevskaja et al thought that Wnt5a in the anaplastic tumor cell is lost but in differentiated thyroid cancers up regulated. ${ }^{53}$ However, McCall et al pointed out that the higher expression of Wnt5a RNA in PTC cells lines (BHP 8-21, BHP 7-13, BHP 2-7 and BHP 10-3) was also examined but in anaplastic or follicular thyroid cancer cells was not observed. ${ }^{54}$ Dong et al found that the expression of Wnt5a was significantly increased in PTC and that the expression of Wnt5a in PTC patients was related to tumor lymph node metastasis as well as TNM stage. ${ }^{55}$

Although Wnt5a and miR-26a-5p are both associated with the occurrence and development of TC, the interrelationship between Wnt5a and miR-26a-5p in PTC is still indistinct. In our study, the expression of miR-26a-5p in PTC tissues and cells was initially examined. It was proved that the expression level of miR-26a-5p in PTC tissues and cells was significantly less than that in normal thyroid tissues and cells. Then, miR-26a-5p could restrain growth, invasion and metastasis of PTC cells that have been demonstrated. Furthermore, the invasion and metastasis of PTC cells were inhibited by miR-26a$5 p$ via Wnt5a as proved. Finally, it was verified that 
Wnt5a was a direct backward position target of microRNA-26a-5p.

In conclusion, our results predicted that miR-26a-5p can target Wnt5a to depress the proliferation, invasion and metastasis of PTC cells. Therefore, accurate treatment of PTC is expected to be achieved.

\section{Acknowledgment}

This work was supported by the Key Research \& Development Planning Guidance Projects of Cangzhou City (No. 172302002).

\section{Disclosure}

The authors report no conflicts of interest in this work.

\section{References}

1. Duan JJ, Yan YQ, Yang NN, et al. International comparison analysis of CHINA'S cancer incidence and mortality. Chin J Front Med Sci. 2016;8:17-23.

2. Khan MS, Pandith AA, Azad N, et al. Impact of molecular alterations of BRAF in the pathogenesis of thyroid cancer. Mutagenesis. 2014;29:131-137. doi:10.1093/mutage/get066

3. Nikiforova MN, Nikiforov YE. Molecular genetics of thyroid cancer: implications for diagnosis, treatment and prognosis. Expert Rev Mol Diagn. 2008;8:83-95. doi:10.1586/14737159.8.1.83

4. Baloch ZW, LiVolsi VA. Special types of thyroid carcinoma Histopathology. 2018;72:40-52. doi:10.1111/his.2018.72.issue-1

5. Regalbuto C, Frasca F, Pellegriti G, et al. Update on thyroid cancer treatment. Future Oncol. 2012;8:1331-1348. doi:10.2217/fon.12.123

6. Oh EM, Lee KE, Kwon H, Kim EY, Bae DS, Youn YK. Analysis of patients with anaplastic thyroid cancer expected to have curative surgery. J Korean Surg Soc. 2012;83:123-129. doi:10.4174/jkss.2012.83.3.123

7. Pemayun TG. Current diagnosis and management of thyroid nodules. Acta Med Indones. 2016;48:247-257.

8. Banerjee M, Muenz DG, Worden FP, Wong SL, Haymart MR. Conditional survival in patients with thyroid cancer. Thyroid. 2014;24:1784-1789. doi:10.1089/thy.2014.0264

9. Ye Y, Song Y, Zhuang J, He S, Ni J, Xia W. Long non-coding RNA CCAL promotes papillary thyroid cancer progression by activation of NOTCH1 pathway. Oncol Res. 2018;26:1383-1390.

10. Liu Z, Lv T, Xie C, Di Z. BRAF V600E gene mutation is associated with bilateral malignancy of papillary thyroid cancer. Am J Med Sci. 2018;356:130-134. doi:10.1016/j.amjms.2018.04.012

11. Wang L, Yao M, Fang M, et al. Expression of hepatic Wnt5a and its clinicopathological features in patients with hepatocellular carcinoma Hepatobiliary Pancreat Dis Int. 2018;17:227-232. doi:10.1016/j. hbpd.2018.03.005

12. Jeon MJ, Kim WG, Choi YM, et al. Features predictive of distant metastasis in papillary thyroid microcarcinoma. Thyroid. 2016;26:161-168. doi:10.1089/thy.2015.0375

13. Lara E, Calvanese V, Huidobro C, et al. Epigenetic repression of ROR2 has a Wnt-mediated, pro-tumourigenic role in colon cancer. Mol Cancer. 2010;9:170. doi:10.1186/1476-4598-9-254

14. Mikels AJ, Nusse R. Purified Wnt5a protein activates or inhibits betacatenin-TCF signaling depending on receptor context. PLoS Biol. 2006;4:e115. doi:10.1371/journal.pbio.0040115

15. McDonald SL, Silver A. The opposing roles of Wnt-5a in cancer. $\mathrm{Br}$ J Cancer. 2009;101:209-214. doi:10.1038/sj.bjc.6605174
16. Wu ZC, Xiong L, Wang LX, et al. Comparative study of ROR2 and WNT5a expression in squamous/adenosquamous carcinoma and adenocarcinoma of the gallbladder. World $J$ Gastroenterol. 2017;23:2601-2612. doi:10.3748/wjg.v23.i14.2601

17. Bartel DP. MicroRNAs: genomics, biogenesis, mechanism, and function. Cell. 2004;116:281-297. doi:10.1016/S0092-8674(04)00045-5

18. Lewis BP, Burge CB, Bartel DP. Conserved seed pairing, often flanked by adenosines, indicates that thousands of human genes are microRNA targets. Cell. 2005;120:15-20. doi:10.1016/j.cell.2004.12.035

19. Ryan BM, Robles AI, Harris CC. Genetic variation in microRNA networks: the implications for cancer research. Nat Rev Cancer. 2010;10:389-402. doi:10.1038/nrc2867

20. Lu J, Getz G, Miska EA, et al. MicroRNA expression profiles classify human cancers. Nature. 2005;435:834-838. doi:10.1038/ nature 03702

21. Dong G, Zhang R, Xu J, Guo Y. Association between microRNA polymorphisms and papillary thyroid cancer susceptibility. Int J Clin Exp Pathol. 2015;8:13450-13457.

22. Gao J, Liu QG. The role of miR-26a-5p in tumors and normal tissues (Review). Oncol Lett. 2011;2:1019-1023. doi:10.3892/ol.2011.413

23. Lu JC, Zhang YP. E2F, HSF2, and miR-26 in thyroid carcinoma: bioinformatic analysis of RNA-sequencing data. Genet Mol Res. 2016;15:15017576.

24. Chen L, Zhao LM, Ding MJ, et al. Higher expression level of tyrosine kinase-like orphan receptor 2 and Wnt member 5a in papillary thyroid carcinoma is associated with poor prognosis. Oncol Lett. 2017;14:5966-5972. doi:10.3892/ol.2017.6989

25. Lee EK, Chung KW, Yang SK, et al. DNA methylation of MAPK signal-inhibiting genes in papillary thyroid carcinoma. Anticancer Res. 2013;33:4833-4839.

26. Ma Y, Qin H, Cui Y. MiR-34a targets GAS1 to promote cell proliferation and inhibit apoptosis in papillary thyroid carcinoma via PI3K/ Akt/Bad pathway. Biochem Biophys Res Commun. 2013;441:958963. doi:10.1016/j.bbrc.2013.11.010

27. Aragon Han P, Weng CH, Khawaja HT, et al. MicroRNA expression and association with clinicopathologic features in papillary thyroid cancer: a systematic review. Thyroid. 2015;25:1322-1329. doi:10.1089/thy.2015.0193

28. Lv XB, Jiao Y, Qing Y, et al. MiR-124 supresses multiple steps of breast cancer metastasis by targeting a cohort of pro-metastatic genes in vitro. Chin J Cancer. 2011;30:821-830. doi:10.5732/cjc.011.10289

29. Pillai RS. MicroRNA function: multiple mechanisms for a tiny RNA? RNA. 2005;11:1753-1761. doi:10.1261/rna.2248605

30. Slack FJ, Weidhaas JB. MicroRNA in cancer prognosis. $N$ Engl $J$ Med. 2008;359:2720-2722. doi:10.1056/NEJMoa0801936

31. Wang V, Wu W. MicroRNA-based therapeutics for cancer. BioDrugs. 2009;23:15-23. doi:10.2165/00063030-200923010-00002

32. Diao Y, Fu H, Wang Q. MiR-221 exacerbate cell proliferation and invasion by targeting TIMP3 in papillary thyroid carcinoma. $\mathrm{Am} \mathrm{J}$ Ther. 2017;24:e317-e328. doi:10.1097/MJT.0000000000000420

33. Zhang X, Li M, Zuo K, et al. Upregulated miR-155 in papillary thyroid carcinoma promotes tumor growth by targeting APC and activating Wnt/ $\beta$-catenin signaling. $J$ Clin Endocrinol Metab. 2013;98:E1305-E1313. doi:10.1210/jc.2012-3602

34. Huang Y, Yu S, Cao S, et al. MicroRNA-222 promotes invasion and metastasis of papillary thyroid cancer through targeting protein phosphatase 2 regulatory subunit $\mathrm{B}$ alpha expression. Thyroid. 2018;28:1162-1173. doi:10.1089/thy.2017.0665

35. Dong S, Meng X, Xue S, Yan Z, Ren P, Liu J. MicroRNA-141 inhibits thyroid cancer cell growth and metastasis by targeting insulin receptor substrate 2. Am J Transl Res. 2016;8:1471-1481.

36. Hua K, Jin J, Zhang H, et al. MicroRNA-7 inhibits proliferation, migration and in vasion of thyroid papillary cancer cells via targeting CKS2. Int J Oncol. 2016;49:1531-1540. doi:10.3892/ijo.2016.3660 
37. Li J, Liang Y, Lv H, et al. MiR-26a-5pa and miR-26a-5pb inhibit esophageal squamous cancer cell proliferation through suppression of c-MYC pathway. Gene. 2017;625:1-9. doi:10.1016/j.gene.2017.05.001

38. Qiu X, Zhu H, Liu S, et al. Expression and prognostic value of microRNA-26a and microRNA-148a in gastric cancer. $J$ Gastroenterol Hepatol. 2017;32:819-827. doi:10.1111/jgh.13533

39. Wang G, Zhang H, He H, et al. Up-regulation of microRNA in bladder tumor tissue is not common. Int Urol Nephrol. 2010;42:95102. doi:10.1007/s11255-009-9584-3

40. Huse JT, Brennan C, Hambardzumyan D, et al. The PTEN-regulating microRNA miR-26a-5pa is amplified in high-grade glioma and facilitates gliomagenesis in vivo. Genes Dev. 2009;23:1327-1337. doi:10.1101/gad.1777409

41. Zhang J, Han C, Wu T. MicroRNA-26a promotes cholangiocarcinoma growth by activating $\beta$-catenin. Gastroenterology. 2012;143:246-256. doi:10.1053/j.gastro.2012.03.045

42. Lv M, Zhang X, Li M, et al. MiR-26a-5pa and its target CKS2 modulate cell growth and tumorigenesis of papillary thyroid carcinoma. PLoS One. 2013;8:e67591. doi:10.1371/journal.pone.0067591

43. Gong Y, Wu W, Zou X, Liu F, Wei T, Zhu J. MiR-26a-5pa inhibits thyroid cancer cell proliferation by targeting ARPP19. Am J Cancer Res. 2018;8:1030-1039.

44. De A. Wnt/Ca2+ signaling pathway: a brief overview. Acta Biochim Biophys Sin. 2011;43:745-756.

45. Wansleeben $C$, Meijlin F. The planar cell polarity pathway in vertebrate development. Dev Dyn. 2011;240:616-626. doi:10.1002/dvdy.22564

46. Nishita M, Enomoto M, Yamagata K, Minami Y. Cell/tissue-tropic functions of Wnt5a signaling in normal and cancer cells. Trends Cell Biol. 2010;20:346-354. doi:10.1016/j.tcb.2010.03.001

47. Dejmek J, Dejmek A, Säfholm A, Sjölander A, Andersson T. Wnt-5a protein expression in primary dukes B colon cancers identifies a subgroup of patients with good prognosis. Cancer Res. 2005;65:9142-9146. doi:10.1158/0008-5472.CAN-05-1710
48. Malgor R, Crouser S, Greco D, et al. Correlation of Wnt5a expression with histopathological grade/stage in urothelial carcinoma of the bladder. Diagn Pathol. 2013;8:139. doi:10.1186/ 1746-1596-8-139

49. Blanc E, Goldschneider D, Douc-Rasy S, Bénard J, Raguénez G. Wnt5a gene expression in malignant human neuroblasts. Cancer Lett. 2005;228:117-123. doi:10.1016/j.canlet.2004.11.061

50. Liang H, Chen Q, Choles AH, et al. Wnt5a inhibits B cell proliferation and functions as a tumor suppressor in hematopoietic tissue. Cancer Cell. 2003;4:349-360.

51. Liu MY, Zhang Y, Zhang J, et al. MicroRNA-1253 suppresses cell proliferation and invasion of non-small-cell lung carcinoma by targeting WNT5A. Cell Death Dis. 2018;9:189. doi:10.1038/ s41419-018-1111-y

52. Zhou H, Zhang ML, Yuan HP, Zheng W, Meng CY, Zhao DX. MicroRNA-154 functions as a tumor suppressor in osteosarcoma by targeting Wnt5a. Oncol Rep. 2016;35:1851-1858. doi:10.3892/ or. 2015.4495

53. Kremenevskaia N, von Wasielewski R, Rao AS, Schöfl C, Andersson T, Brabant G. Wnt-5a has tumor suppressor activity in thyroid carcinoma. Oncogene. 2005;24:2144-2154. doi:10.1038/sj.onc. 1208370

54. McCall KD, Harii N, Lewis CJ, et al. High basal levels of functional toll-like receptor 3(TLR3) and noncanonical Wnt5a are expressed in papillary thyroid cancer and are coordinately decreased by phenylmethimazole together with cell proliferation and migration. Endocrinology. 2007;148:4226-4237. doi:10.1210/en.2007-0459

55. Dong Y, Shi JX, Yan L, Wang YH, Wang L, Shi QB. Wnt5a expression in thyroid papillary carcinoma and its significance. Chin J Gen Surg. 2013;22:1479-1483.

\section{Publish your work in this journal}

OncoTargets and Therapy is an international, peer-reviewed, open access journal focusing on the pathological basis of all cancers, potential targets for therapy and treatment protocols employed to improve the management of cancer patients. The journal also focuses on the impact of management programs and new therapeutic agents and protocols on patient perspectives such as quality of life, adherence and satisfaction. The manuscript management system is completely online and includes a very quick and fair peer-review system, which is all easy to use. Visit http://www.dovepress.com/ testimonials.php to read real quotes from published authors. 